\title{
INTEGRAL EQUATIONS OF THE FIRST KIND OF SONINE TYPE
}

\author{
STEFAN G. SAMKO and ROGÉRIO P. CARDOSO
}

Received 27 November 2002

\begin{abstract}
A Volterra integral equation of the first kind $K \varphi(x): \equiv \int_{-\infty}^{x} k(x-t) \varphi(t) d t=f(x)$ with a locally integrable kernel $k(x) \in L_{1}^{\text {loc }}\left(\mathbb{R}_{+}^{1}\right)$ is called Sonine equation if there exists another locally integrable kernel $\ell(x)$ such that $\int_{0}^{x} k(x-t) \ell(t) d t \equiv 1$ (locally integrable divisors of the unit, with respect to the operation of convolution). The formal inversion $\varphi(x)=(d / d x) \int_{0}^{x} \ell(x-t) f(t) d t$ is well known, but it does not work, for example, on solutions in the spaces $X=L_{p}\left(\mathbb{R}^{1}\right)$ and is not defined on the whole range $K(X)$. We develop many properties of Sonine kernels which allow us-in a very general case-to construct the real inverse operator, within the framework of the spaces $L_{p}\left(\mathbb{R}^{1}\right)$, in Marchaud form: $K^{-1} f(x)=$ $\ell(\infty) f(x)+\int_{0}^{\infty} \ell^{\prime}(t)[f(x-t)-f(x)] d t$ with the interpretation of the convergence of this "hypersingular" integral in $L_{p}$-norm. The description of the range $K(X)$ is given; it already requires the language of Orlicz spaces even in the case when $X$ is the Lebesgue space $L_{p}\left(\mathbb{R}^{1}\right)$.
\end{abstract}

2000 Mathematics Subject Classification: 45D05, 45H05, 44A15, 26 A33.

1. Introduction. We study integral equations of the first kind on the real axis of the form

$$
\mathbf{K} \varphi:=\int_{-\infty}^{x} k(x-t) \varphi(t) d t=f(x), \quad x \in \mathbb{R}
$$

where the kernel $k(x)$ is assumed to satisfy the condition that there exists another kernel $\ell(x)$ such that

$$
\int_{0}^{x} \ell(x-t) k(t) d t=1, \quad x>0
$$

the latter condition being known as the Sonine condition due to Sonine [8], where such equations were firstly considered in the case of finite interval, see [7, page 85], about Sonine equations.

A well-known particular case of the Sonine kernel is the kernel of fractional integration

$$
k(x)=\frac{x^{\alpha-1}}{\Gamma(\alpha)}, \quad x>0,0<\alpha<1
$$


for which $\ell(x)=x^{-\alpha} / \Gamma(1-\alpha)$, see [7, Section 2]. Other examples can be found in Section 7 . There exist many examples of Sonine equations with special functions in the kernel, see Section 7; such equations arise in many applications.

\section{Preliminaries}

\subsection{Definitions}

DEFINITION 2.1. A kernel $k(x) \in L_{1}^{\text {loc }}\left(\mathbb{R}_{+}^{1}\right)$ is called a Sonine kernel if there exists a kernel $\ell(x) \in L_{1}^{\text {loc }}\left(\mathbb{R}_{+}^{1}\right)$ such that relation (1.2) is valid for almost all $x \in \mathbb{R}_{+}^{1}$. Correspondingly, the operator $\mathbf{K}$ with a Sonine kernel $k(x)$ will be called Sonine integral operator.

The kernel $\ell(x)$ will be referred to as the kernel associated to the kernel $k(x)$. Obviously, $\ell(x)$ is also a Sonine kernel.

From (1.2) it follows that both the Sonine kernel $k(x)$ and its associate $\ell(x)$ are necessarily unbounded as $x \rightarrow 0$.

In the case where the kernels are both monotonous of the same sense, the following Chebyshev inequality is valid:

$$
\int_{0}^{x} \ell(x-t) k(t) d t \leq \frac{1}{x} \int_{0}^{x} k(t) d t \int_{0}^{x} \ell(t) d t, \quad x>0 .
$$

We observe that the standard proof of the Chebyshev inequality (2.1) uses the assumption that the product $k(t) \ell(t)$ is integrable, which is not the case for Sonine kernels; however, the Chebyshev inequality may be proved without that assumption, see the appendix.

2.2. On identity approximations. An operator $A_{\varepsilon}$ is said to be an identity approximation in a Banach space $X$ if $\left\|A_{\varepsilon} f-f\right\|_{X} \rightarrow 0$ as $\varepsilon \rightarrow 0$. Let $A_{\varepsilon}$ be a convolution operator

$$
A_{\varepsilon} \varphi=\int_{\mathbb{R}^{n}} a_{\varepsilon}(t) \varphi(x-t) d t, \quad \varepsilon>0 .
$$

The following statement on sufficient conditions for operator (2.2) to be an identity approximation is well known at least for $L_{p}$-spaces. For completeness, we give it with the proof in the following general form, see Lemma 2.3, in which $X=X\left(\mathbb{R}^{n}\right)$ is an arbitrary space of functions defined on $\mathbb{R}^{n}$, satisfying the following axioms:

(1) $X$ is translation invariant: $\|f(x-h)\|_{X} \leq C\|f\|_{X}$ with $C$ not depending on $h \in \mathbb{R}^{n}$ and the mean continuity with respect to the norm $\|\cdot\|_{X}$ holds

$$
\omega_{X}(f, \delta):=\sup _{|h|<\delta}\|f(x-h)-f(x)\|_{X} \longrightarrow 0 \quad \text { as } \delta \longrightarrow 0 ;
$$

(2) Minkowsky integral inequality is valid

$$
\left\|\int_{\mathbb{R}^{n}} f(\cdot, t) d t\right\|_{X} \leq \int_{\mathbb{R}^{n}}\|f(\cdot, t)\|_{X} d t .
$$


REMARK 2.2. The above assumptions (1) and (2) obviously hold for the spaces $L_{p}\left(\mathbb{R}^{n}\right), 1 \leq p<\infty$, and also for the Orlicz spaces $L_{\Phi}\left(\mathbb{R}^{n}\right)$ with a Young function $\Phi(u)$ satisfying the $\Delta_{2}$-condition: $\Phi(2 x) \leq k \Phi(x)$ for large values of $x$, with some $k>0$; the validity of Minkowsky integral inequality is in fact a consequence of the definition of the Orlicz norm and does not need the $\Delta_{2}$ condition, while $\Phi$-mean continuity requires this condition, see [1, page 179]. See also [1, page 192], about identity approximation with dilatation invariant kernel in the case of the Orlicz space $E_{\Phi}$.

LEMMA 2.3. Let a Banach function space $X$ satisfy assumptions (1) and (2) and functions $a_{\varepsilon}(t)$ satisfy the conditions

$$
\lim _{\varepsilon \rightarrow 0} \int_{\mathbb{R}^{n}} a_{\varepsilon}(t) d t=1, \quad \int_{\mathbb{R}^{n}}\left|a_{\varepsilon}(t)\right| d t \leq M<\infty
$$

with $M$ not depending on $\varepsilon$ and

$$
\lim _{\varepsilon \rightarrow 0} \int_{|t| \geq \delta}\left|a_{\varepsilon}(t)\right| d t=0
$$

for any $\delta>0$. Then $A_{\varepsilon}$ is an identity approximation in the space $X$ and for any $\delta>0$ the estimate

$$
\left\|A_{\varepsilon} f-f\right\|_{X} \leq C(\varepsilon, \delta)\|f\|_{X}+M \omega_{X}(f, \delta)
$$

is valid where

$$
C(\varepsilon, \delta)=\left|\int_{R^{n}} a_{\varepsilon}(x) d x-1\right|+2 \int_{|x|>\delta}\left|a_{\varepsilon}(x)\right| d x .
$$

In particular, in the case $a_{\varepsilon}(x)=\left(1 / \varepsilon^{n}\right) a(x / \varepsilon)$, estimate (2.7) reduces to

$$
\left\|A_{\varepsilon} f-f\right\|_{X} \leq C(\varepsilon)\|f\|_{X}+M \omega_{X}\left(f, \varepsilon \ln \frac{1}{\varepsilon}\right)
$$

with

$$
C(\varepsilon)=2 \int_{|x|>\ln (1 / \varepsilon)}|a(x)| d x, \quad M=\int_{R^{n}}|a(x)| d x .
$$

Proof. We have

$$
\begin{aligned}
\left\|A_{\varepsilon} f-f\right\|_{X} \leq & \left\|\int_{\mathbb{R}^{n}} a_{\varepsilon}(t)[f(x-t)-f(x)] d t\right\|_{X} \\
& +\left|\int_{\mathbb{R}^{n}} a_{\varepsilon}(t) d t-1\right|\|f\|_{X} \\
& =C_{\varepsilon}+D_{\varepsilon} .
\end{aligned}
$$


By the first condition in (2.5), the term $D_{\varepsilon}$ in (2.11) vanishes as $\varepsilon \rightarrow 0$. For the term $C_{\varepsilon}$ we have

$$
\begin{aligned}
C_{\varepsilon} \leq & \left\|\int_{|t|<\delta} a_{\varepsilon}(t)[f(x-t)-f(x)] d t\right\|_{X} \\
& +\left\|\int_{|t|>\delta} a_{\varepsilon}(t)[f(x-t)-f(x)] d t\right\|_{X} \\
= & E_{\varepsilon}^{\delta}+F_{\varepsilon}^{\delta} .
\end{aligned}
$$

By Minkowsky inequality (2.4) and translation invariance, we obtain

$$
\begin{aligned}
F_{\varepsilon}^{\delta} & \leq \int_{|t|>\delta}\left|a_{\varepsilon}(t)\right|\|f(x-t)-f(x)\|_{X} d t \\
& \leq(1+C)\|f\|_{X} \int_{|t|>\delta}\left|a_{\varepsilon}(t)\right| d t
\end{aligned}
$$

which tends to 0 as $\varepsilon \rightarrow 0$ by (2.6). For $E_{\varepsilon}^{\delta}$ we have

$$
E_{\varepsilon}^{\delta} \leq \sup _{|t|<\delta}\|f(x-t)-f(x)\|_{X} \int_{0}^{\infty}\left|a_{\varepsilon}(t)\right| d t \leq M \omega_{X}(f, \delta)
$$

by the second condition in (2.5).

Gathering the estimates, we arrive at (2.7) which yields the convergence by arbitrariness of $\delta$. To obtain (2.9), it suffices to choose $\delta=\varepsilon \ln (1 / \varepsilon)$.

2.3. About $L_{p}$-setting for the Sonine equation. To consider the Sonine operator on $L_{p}\left(\mathbb{R}^{1}\right)$, we have to introduce some condition on the kernel, guaranteeing the existence of the integral on $L_{p}$-functions. Obviously, if $k(x)$ has a special form

$$
k(x)=\frac{m(x)}{x^{1-\alpha}}, \quad x>0, \sup _{x>0}|m(x)|<\infty,
$$

with a bounded function $m(x)$ and some $\alpha \in(0,1)$, then a sufficient condition is $\alpha<1 / p$ by the Hardy-Littlewood theorem for fractional integrals. In this case the operator acts from $L_{p}\left(\mathbb{R}^{1}\right), 1<p<1 / \alpha$, into $L_{q}\left(\mathbb{R}^{1}\right), 1 / q=1 / p-\alpha$. For an arbitrary Sonine kernel, the range already does not belong to any $L_{q}\left(\mathbb{R}^{1}\right)$, but may be embedded into some Orlicz space according to Theorem 2.4.

First we note that a convolution with a locally integrable kernel $k(x)$ is well defined for any function $f \in L_{p}\left(\mathbb{R}^{1}\right)$ if there exists an $N>0$ such that

$$
\int_{N}^{\infty}|k(x)|^{p^{\prime}} d x<\infty, \quad \frac{1}{p}+\frac{1}{p^{\prime}}=1 .
$$

Indeed,

$$
\int_{0}^{\infty} k(t) f(x-t) d t=\int_{0}^{N} k(t) f(x-t) d t+\int_{N}^{\infty} k(t) f(x-t) d t,
$$


where the first term exists almost everywhere by the Minkowsky inequality and the second term is even bounded by the Hölder inequality.

In Theorem 2.4 we use the notation

$$
k^{* *}(t)=\frac{1}{t} \int_{0}^{t} k^{*}(s) d s,
$$

where $k^{*}(s)=\inf \{t>0: m(k, t) \leq s\}$ is the nonincreasing rearrangement of $|k(x)|$, inverse to the distribution function $m(k, t)=\operatorname{mes}\left\{x \in \mathbb{R}^{1}:|k(x)|>t\right\}$. Observe that

$$
k^{* *}(t) \geq k^{*}(t), \quad\left\|k^{*}\right\|_{p}=\|k\|_{p}, \quad 1 \leq p<\infty .
$$

Also

$$
\left\|k^{* *}\right\|_{p} \leq p^{\prime}\|k\|_{p}, \quad 1<p<\infty
$$

by boundedness of the Hardy operator in $L_{p}$.

The following statement is a particular case of the O'Neil theorem on boundedness of convolution operators in Orlicz spaces, see [2, page 316].

THEOREM 2.4. Let $k(x) \in L_{1}^{\text {loc }}\left(\mathbb{R}^{1}\right)$. The convolution operator $\mathbf{K}$ is defined for all $\varphi \in L_{p}\left(\mathbb{R}^{1}\right), 1<p<\infty$, under condition (2.16) or the condition

$$
\int_{N}^{\infty} \frac{\left|k^{* *}(x)\right|}{x^{1 / p}} d x<\infty
$$

with some $N>0$. Under condition (2.21) the operator $\mathbf{K}$ is bounded from $L_{p}\left(\mathbb{R}^{1}\right)$ into the Orlicz space $L_{\Phi}\left(\mathbb{R}^{1}\right)$ with the Young function $\Phi(x)$ defined by its inverse

$$
\Phi^{-1}(x)=\int_{1 / x}^{\infty} \frac{k^{* *}(t)}{t^{1 / p}} d t=p\left[x^{1 / p} \int_{0}^{1 / x}|k(s)| d s+\int_{1 / x}^{\infty} \frac{k^{*}(s)}{s^{1 / p}} d s\right] .
$$

REMARK 2.5. The condition

$$
\int_{N}^{\infty}|k(x)|^{r} d x<\infty
$$

for some $r<p^{\prime}$ is sufficient for the validity of (2.21) which is easily obtained by the Hölder inequality and properties (2.19) and (2.20).

REMARK 2.6. From (2.22), by the direct differentiation we also have

$$
\frac{d \Phi^{-1}(x)}{d x}=\frac{k^{* *}(1 / x)+p\left[k^{*}(1 / x)-|k(1 / x)|\right]}{x^{2-1 / p}} .
$$

3. Sonine equation in $L_{1}(-\infty, b), b \leq+\infty$. Let

$$
\mathbf{L} f:=\int_{-\infty}^{x} \ell(x-t) f(t) d t
$$


where $\ell(x)$ is the kernel associated with $k(x)$. Formally applying the operator $\mathbf{L}$ to the operator $\mathbf{K}$ from the left, we arrive at the relation

$$
\int_{-\infty}^{x} \varphi(s) d s=\int_{-\infty}^{x} l(x-t) f(t) d t
$$

due to condition (1.2). Then the formal solution of (1.1) is

$$
\varphi(x)=\mathbf{K}^{-1} f:=\frac{d}{d x} \int_{-\infty}^{x} l(x-t) f(t) d t .
$$

To justify this inversion for solutions in $L_{1}(-\infty, b)$, we recall the following definition, see [7, page 130].

DEFINITION 3.1. A function $g(x)$ is said to be absolutely continuous on $(-\infty, b): g(x) \in \mathrm{AC}(-\infty, b)$ if it is absolutely continuous on every finite interval and has bounded variation on $[-\infty, b]$.

The condition $g(x) \in \mathrm{AC}(-\infty, b)$ is equivalent to the representation of $g(x)$ in the form

$$
g(x)=\int_{-\infty}^{x} \varphi(t) d t+\text { const } \quad \text { with } \varphi(x) \in L_{1}(-\infty, b) .
$$

The following statement is valid.

TheOREM 3.2. Let $k(x)$ be a Sonine kernel. Equation (1.1) is solvable in $L_{1}(-\infty, b)$ if and only if the function $g_{\ell}(x):=\int_{-\infty}^{x} l(x-t) f(t) d t$ satisfies the conditions

$$
g_{\ell}(x) \in \mathrm{AC}(-\infty, b), \quad g_{\ell}(-\infty)=0 .
$$

Under these conditions the equation has a unique solution given by (3.3).

The proof of Theorem 3.2 is similar to the case of kernel (1.3) (see [7, Theorem 2.1, page 31 and Theorem 6.3, page 131]).

The following lemma, in which

$$
L(x)=\int_{0}^{x} \ell(s) d s
$$

is the primitive of the kernel $\ell(s)$, gives a sufficient condition for validity of (3.5) in terms of the function $f$ itself.

LEMMA 3.3. If (a) $f(x) \in \mathrm{AC}(-\infty, b), f(-\infty)=0$, (b) $\int_{-\infty}^{x} f^{\prime}(t) \ell(x-t) d t \in$ $L^{1}(-\infty, b)$, (c) the integral $\int_{-\infty}^{x} f^{\prime}(s) L(x-s) d s$ converges for all $x \in(-\infty, b)$, then $g_{\ell}(x) \in \mathrm{AC}(-\infty, b)$ and $g_{\alpha}(-\infty)=0$ and $g_{\alpha}(x)$ may also be represented as

$$
g_{\ell}(x)=\int_{-\infty}^{x} f^{\prime}(s) L(x-s) d s .
$$


Proof. By condition (a) of the lemma, we have

$$
g_{\ell}(x)=\int_{-\infty}^{x} l(x-t) d t \int_{-\infty}^{t} f^{\prime}(s) d s
$$

whence (3.7) follows after the interchange of the order of integration, this interchange being easily justified by condition (c).

To obtain (3.7), we observe that the following relation holds:

$$
\int_{-\infty}^{x} \ell(x-t) d t \int_{-\infty}^{t} f^{\prime}(s) d s=\int_{-\infty}^{x}\left(\int_{-\infty}^{t} f^{\prime}(s) \ell(t-s) d s\right) d t
$$

which may be verified via the interchange of the order of integration. Then the statements $g_{\ell}(x) \in \mathrm{AC}(-\infty, b)$ and $g_{\ell}(-\infty)=0$ follow from condition (b) of the lemma.

\section{Properties of Sonine kernels}

LEMMA 4.1. Let a Sonine kernel $k(x)$ and its associate $\ell(x)$ have locally integrable derivatives and satisfy the conditions

$$
\lim _{t \rightarrow 0} t k(t)=0, \quad \lim _{t \rightarrow 0} t \ell(t)=0
$$

Then the formula

$$
\int_{0}^{x} \ell^{\prime}(t)[k(x)-k(x-t)] d t=k(x) \ell(x)
$$

is valid for almost all $x>0$ (for all $x>0$ if $k^{\prime}(x)$ and $\ell^{\prime}(x)$ exist at every point $x>0)$.

Proof. First we observe that from (4.1) it follows that the integrals $\int_{0}^{1} t k^{\prime}(t) d t$ and $\int_{0}^{1} t \ell^{\prime}(t) d t$ exist (integration by parts). From (1.2) we have

$$
x \int_{0}^{1} \ell(x-x t) k(x t) d t=1 .
$$

Differentiating with respect to $x$, we obtain

$$
\begin{gathered}
x \int_{0}^{1}\left[\ell^{\prime}(x-x t)(1-t) k(x t)+\ell(x-x t) k^{\prime}(x t) t\right] d t \\
+\int_{0}^{1} \ell(x-x t) k(x t) d t=0 .
\end{gathered}
$$

After the inverse change of variables we have

$$
\begin{gathered}
\int_{0}^{x} \ell^{\prime}(x-t)(x-t) k(t) d t+\int_{0}^{x} \ell(x-t) k^{\prime}(t) t d t \\
+\int_{0}^{x} \ell(x-t) k(t) d t=0 .
\end{gathered}
$$


Here the third term is identically equal to 1 . In the second term we represent $k^{\prime}(t) d t$ as $d[k(t)-k(x)]$ and integrate by parts. Nonintegral terms vanish because of (4.1) and the fact that $\lim _{t \rightarrow 0} \ell(t)[k(x)-k(x-t)]=0$ for almost all $x>0$ which follows from (4.1) and existence of the $\operatorname{limit}_{t \rightarrow 0}((k(x)-k(x-$ $t)) / t$ ) for almost all $x>0$. As a result we reduce the above relation to

$$
\int_{0}^{x} \ell^{\prime}(x-t)[x k(t)-t k(x)] d t+k(x) \int_{0}^{x} \ell(t) d t=0
$$

Hence

$$
x \int_{0}^{x} \ell^{\prime}(t)[k(x)-k(x-t)] d t=k(x) \int_{0}^{x}\left[\ell^{\prime}(t) t+\ell(t)\right] d t
$$

which yields (4.2) since $\ell^{\prime}(t) t+\ell(t)=(d / d t)[t \ell(t)]$.

REMARK 4.2. In Lemma 4.3 we show that condition (4.1) is satisfied automatically for both $k(x)$ and $\ell(x)$ if $k(x)$ and $\ell(x)$ are nonincreasing in a neighborhood of the origin.

The inversion of the Sonine equation for $L_{p}$-solutions will be realized under some additional assumptions on the kernel $k(x)$ and its associate $\ell(x)$.

MONOTONICITY NEAR THE ORIGIN. There exists a neighborhood $0<x<\varepsilon_{0}$ where

$$
k(x) \geq 0, \quad \ell(x) \geq 0, \quad k(x) \downarrow, \quad \ell(x) \downarrow, \quad 0<x \leq \varepsilon_{0} .
$$

Absolute integrability of $k^{\prime}(x)$ AND $\ell^{\prime}(x)$ AT INFinity. It is assumed that derivatives exist in the generalized sense and are locally integrable and

$$
\int_{1}^{\infty}\left|k^{\prime}(x)\right| d x<\infty, \quad \int_{1}^{\infty}\left|\ell^{\prime}(x)\right| d x<\infty
$$

Note that a kernel satisfying condition (4.9) stabilizes at infinity

$$
\exists \lim _{x \rightarrow \infty} k(x)=k(\infty), \quad|k(\infty)|<\infty .
$$

Based on the above additional assumptions (4.8) and (4.9), one may derive many special properties of $k(x)$ from the condition for $k(x)$ to be Sonine kernel, such as its behavior as $x \rightarrow 0$ and $x \rightarrow \infty$ and various integral estimates. They are summarized in the following two lemmas which are crucial for further proofs. 
LEMMA 4.3. Any Sonine kernel satisfying assumption (4.8) has the following properties:

$$
\begin{aligned}
& x k(x) \ell(x) \leq 1, \quad 0<x \leq \varepsilon_{0}, \\
& k(x) \int_{0}^{x} l(t) d t \leq 1, \quad \ell(x) \int_{0}^{x} k(t) d t \leq 1, \quad 0<x \leq \varepsilon_{0}, \\
& k(x) \int_{0}^{x} l(t) d t+\ell(x) \int_{0}^{x} k(t) d t \geq 1, \quad 0<x \leq \varepsilon_{0}, \\
& \int_{0}^{x} k(t) d t \int_{0}^{x} \ell(t) d t \geq x, \quad 0<x \leq \varepsilon_{0}, \\
& \lim _{x \rightarrow 0+} k(x)=\lim _{x \rightarrow 0+} \ell(x)=+\infty, \\
& \lim _{x \rightarrow 0+} x k(x)=\lim _{x \rightarrow 0+} x \ell(x)=0, \\
& \sup _{0<\varepsilon<\varepsilon_{0}}\left[\int_{0}^{\varepsilon}\left|k^{\prime}(t)\right| d t \int_{\varepsilon-t}^{\varepsilon} l(s) d s\right] \leq 1 .
\end{aligned}
$$

Proof. By the monotonicity condition (4.8), in (1.2) we have $k(t) \geq k(x)$, $\ell(x-t) \geq \ell(x), 0<x \leq \varepsilon_{0}$. Therefore,

$$
\int_{0}^{x} \ell(x-t) k(t) d t \geq \ell(x) \int_{0}^{x} k(t) d t \geq \ell(x) k(x) x
$$

so that properties (4.11) and (4.12) follow immediately from (1.2).

Inequality (4.13) is proved as follows:

$$
\begin{aligned}
1 & \equiv \int_{0}^{x} k(t) \ell(2 x-t) d t+\int_{x}^{2 x} k(t) \ell(2 x-t) d t \\
& \leq \ell(x) \int_{0}^{x} k(t) d t+k(x) \int_{x}^{2 x} \ell(2 x-t) d t .
\end{aligned}
$$

Inequality (4.14) is a consequence of the Sonine condition (1.2) and Chebyshev inequality (2.1).

Property (4.15) follows from (4.12) by monotonicity of the functions $k(x)$ and $\ell(x)$ near the origin. Taking (4.15) into account, we see that (4.16) is a consequence of (4.11).

To check (4.17), we observe that

$$
\begin{aligned}
\int_{0}^{\varepsilon}\left|k^{\prime}(t)\right| d t \int_{\varepsilon-t}^{\varepsilon} \ell(s) d s & =-\int_{0}^{\varepsilon} \ell(s) d s \int_{\varepsilon-s}^{\varepsilon} k^{\prime}(t) d t \\
& =\int_{0}^{\varepsilon} \ell(s)[k(\varepsilon-s)-k(\varepsilon)] d s \\
& =1-k(\varepsilon) \int_{0}^{\varepsilon} \ell(s) d s \leq 1
\end{aligned}
$$

with (1.2) taken into account. 
LEMMA 4.4. If a Sonine kernel $k(x)$ satisfies the integrability condition (4.9) and $k(\infty)=0$, then

$$
\begin{gathered}
\lim _{N \rightarrow \infty} \int_{N}^{N+a} k(t) d t=0 \quad \text { for any } a>0, \\
\int_{0}^{\varepsilon} k(t) d t \leq \int_{0}^{\infty}|k(t+\varepsilon)-k(t)| d t, \quad 0<\varepsilon<\varepsilon_{0} .
\end{gathered}
$$

If $k(x)$ satisfies both (4.9) and (4.8), then

$$
\int_{0}^{\infty}|k(x+h)-k(x)| d x<\infty
$$

for any $h \in \mathbb{R}^{1}$ and

$$
\sup _{0<\varepsilon<\varepsilon_{0}}\left[\ell(\varepsilon) \int_{0}^{\infty}|k(x+\varepsilon)-k(x)| d x\right]<\infty,
$$

and also

$$
\sup _{0<\varepsilon<\varepsilon_{0}}\left[\int_{0}^{\varepsilon} \ell(t) d t \int_{\varepsilon}^{\infty}\left|k^{\prime}(t)\right| d t\right]<\infty
$$

PROoF. First we prove (4.21). This property is derived from the inequality

$$
\left|\int_{N}^{N+a} k(s) d s\right| \leq a \int_{N}^{\infty}\left|k^{\prime}(t)\right| d t
$$

and condition (4.9) for $k(x)$; inequality (4.26) is derived from the equality

$$
\int_{N}^{N+a} k(s) d s=-\int_{N}^{N+a} d s \int_{s}^{\infty} k^{\prime}(t) d t
$$

To prove (4.23), let $h>0$. Obviously

$$
\begin{aligned}
I_{h} & :=\int_{0}^{\infty}|k(x+h)-k(x)| d x=\int_{0}^{\infty} d x\left|\int_{0}^{h} k^{\prime}(x+t) d t\right| \\
& \leq \int_{0}^{h} d t \int_{t}^{\infty}\left|k^{\prime}(x)\right| d x .
\end{aligned}
$$

Let $h \geq \varepsilon_{0}$ (the case $h \leq \varepsilon_{0}$ is easier). We have

$$
\begin{aligned}
I_{h} & \leq \int_{0}^{h} d t\left(\int_{t}^{\varepsilon_{0}}+\int_{\varepsilon_{0}}^{\infty}\right)\left|k^{\prime}(x)\right| d x \\
& =h \int_{\varepsilon_{0}}^{\infty}\left|k^{\prime}(x)\right| d x-\int_{0}^{h} d t \int_{0}^{\varepsilon_{0}} k^{\prime}(x) d x \\
& \leq h \int_{\varepsilon_{0}}^{\infty}\left|k^{\prime}(x)\right| d x+\int_{0}^{h} k(t) d t
\end{aligned}
$$

which proves (4.23). 
To prove property (4.24), we follow the same line as above with $h=\varepsilon$ : multiply (4.29) by $\ell(\varepsilon)$ and make use of properties (4.12) and (4.16).

To prove (4.25), we proceed similarly to actions in (4.29) and obtain

$$
\int_{0}^{\varepsilon} \ell(t) d t \int_{\varepsilon}^{\infty}\left|k^{\prime}(t)\right| d t=-\int_{0}^{\varepsilon} \ell(t) d t \int_{\varepsilon}^{\varepsilon_{0}} k^{\prime}(t) d t+c \int_{0}^{\varepsilon} \ell(t) d t
$$

where $c=\int_{\varepsilon_{0}}^{\infty}\left|k^{\prime}(t)\right| d t$. Hence

$$
\int_{0}^{\varepsilon} \ell(t) d t \int_{\varepsilon}^{\infty}\left|k^{\prime}(t)\right| d t=\left[k(\varepsilon)-k\left(\varepsilon_{0}\right)\right] \int_{0}^{\varepsilon} \ell(t) d t+c \int_{0}^{\varepsilon} \ell(t) d t
$$

so that, to get (4.25), it suffices to refer to property (4.12).

Finally,

$$
\begin{aligned}
\int_{0}^{\varepsilon} k(t) d t & =\int_{0}^{N}|k(t)| d t-\int_{\varepsilon}^{N}|k(t)| d t \\
& =\int_{0}^{N-\varepsilon}\{|k(t)|-|k(t+\varepsilon)|\} d t+\int_{N-\varepsilon}^{N}|k(t)| d t .
\end{aligned}
$$

Making use of property (4.21) as $N \rightarrow \infty$, we arrive at (4.22).

\section{The Marchaud form of the inverse operator}

5.1. The Marchaud-type construction on "nice" functions. In the theory of fractional differentiation it is known that the Liouville fractional differentiation

$$
\mathscr{D}^{\alpha} f=\frac{1}{\Gamma(1-\alpha)} \frac{d}{d x} \int_{-\infty}^{x} \frac{f(t) d t}{(x-t)^{\alpha}}, \quad 0<\alpha<1,
$$

may be represented in the form

$$
\mathscr{D}^{\alpha} f=\frac{\alpha}{\Gamma(1-\alpha)} \int_{0}^{\infty} \frac{f(x)-f(x-t)}{t^{1+\alpha}} d t
$$

known as Marchaud fractional derivative, see [7, page 109]. This form is much more appropriate for the inversion of fractional integration within the frameworks of $L_{p}$-spaces than the Liouville form, see [7, page 123]. Our goal is to invert the operator $\mathbf{K}$ with an arbitrary Sonine kernel in $L_{p}\left(\mathbb{R}^{1}\right)$, so our first aim is to find a corresponding analogue of the representation (5.2).

LEMMA 5.1. Let the associate Sonine kernel $\ell(t)$ satisfy the condition

$$
\lim _{x \rightarrow 0} x \ell(x)=0
$$

and have derivative $\ell^{\prime}(x) \in L_{1}(\varepsilon, N)$ for all $0<\varepsilon<N<\infty$. Then operator (3.3) may be represented in the form

$$
\left(\mathbf{K}^{-1} f\right)(x)=\ell(\infty) f(x)+\int_{0}^{\infty}[f(x-t)-f(x)] \ell^{\prime}(t) d t
$$


at least on "nice" functions $f(x)$ for which the integral converges, for example, on functions $f(x) \in C_{0}^{\infty}\left(\mathbb{R}^{1}\right)$.

Proof. First we observe that $\ell(\infty)$ exists by (4.9), as in (4.10). For "nice" function $f$ we have

$$
\left(\mathbf{K}^{-1} f\right)(x)=\int_{0}^{\infty} \ell(t) f^{\prime}(x-t) d t=\lim _{N \rightarrow \infty} \int_{0}^{N} \ell(t) f^{\prime}(x-t) d t .
$$

We substitute $\ell(t)=\ell(N)-\int_{t}^{N} \ell^{\prime}(\xi) d \xi$, integrate by parts, and arrive at (5.4). Since $f(x-t)-f(x) \sim c t$ as $t \rightarrow 0$ for "nice" functions, convergence of the integral on the right-hand side of (5.4) is seen by integrating by parts due to the condition $\lim _{x \rightarrow 0} x \ell(x)=0$.

When inverting the operator $\mathbf{K}$ under the $L_{p}$-setting, we will interpret operator (5.4) as the limit of the corresponding truncated integrals

$$
\left(\mathbf{K}^{-1} f\right)(x)=\lim _{\varepsilon \rightarrow 0^{+}}\left(\mathbf{K}_{\varepsilon}^{-1} f\right)(x)
$$

with

$$
\left(\mathbf{K}_{\varepsilon}^{-1} f\right)(x)=\ell(\infty) f(x)+\int_{\varepsilon}^{\infty}[f(x-t)-f(x)] \ell^{\prime}(t) d t
$$

and we will prove the general inversion statement $\mathbf{K}^{-1} \mathbf{K} \varphi=\varphi, \varphi \in L_{p}\left(\mathbb{R}^{1}\right)$, for an arbitrary Sonine kernel satisfying rather general assumptions, with the limit in (5.6) treated in $L_{p}$-norm.

The operator $\mathbf{K}_{\varepsilon}^{-1}$ will be referred to as the truncated Marchaud-Sonine operator.

\subsection{Integral representation of the truncated Marchaud-Sonine operator}

\subsubsection{The composition $\mathrm{K}_{\varepsilon}^{-1} \mathrm{~K}$}

THEOREM 5.2. Let a Sonine kernel $k(x)$ and its associate $\ell(x)$ satisfy conditions (2.21), (4.9), and (4.8). Then the truncated Marchaud-Sonine operator on functions $f(x)=\mathbf{K} \varphi$ with $\varphi(x) \in L_{p}\left(\mathbb{R}^{n}\right), 1 \leq p<\infty$, has the following representation:

$$
\left(\mathbf{K}_{\varepsilon}^{-1} \mathbf{K} \varphi\right)(x)=\int_{0}^{\infty} N_{\varepsilon}(t) \varphi(x-t) d t
$$

where

$$
N_{\varepsilon}(s)=\int_{\varepsilon}^{\infty} \ell^{\prime}(t)\left[k_{+}(s-t)-k(s)\right] d t+\ell(\infty) k(s) .
$$

Proof. First, we note that $\mathbf{K} \varphi(x)$ exists almost everywhere in the case $\varphi(x) \in L_{p}\left(\mathbb{R}^{n}\right)$, by Theorem 2.4. Then $\mathbf{K}_{\varepsilon}^{-1} \mathbf{K} \varphi(x)$ exists almost everywhere as 
a convolution of a function in Orlicz space with an integrable kernel $\ell^{\prime}(x) \in$ $L_{1}(\varepsilon, \infty)$, the latter being well known and following for example from (2.4), see Remark 2.2.

We have

$$
f(x-t)-f(x)=\int_{0}^{\infty}\left[k_{+}(s-t)-k(s)\right] \varphi(x-s) d s,
$$

where

$$
k_{+}(s)= \begin{cases}k(s), & s>0 \\ 0, & s<0\end{cases}
$$

Then

$$
\left(\mathbf{K}_{\varepsilon}^{-1} f\right)(x)=\int_{\varepsilon}^{\infty}\left[\int_{0}^{\infty}\left[k_{+}(s-t)-k(s)\right] \varphi(x-s) d s\right] \ell^{\prime}(t) d t+\ell(\infty)(\mathbf{K} \varphi)(x)
$$

and after the interchange of order of integration we arrive at (5.8) and (5.9).

It remains to justify the above interchange. By Fubini theorem it suffices to show that the integral

$$
I(x):=\int_{0}^{\infty}|\varphi(x-s)| d s \int_{\varepsilon}^{\infty}\left|\ell^{\prime}(t)\right| \cdot\left|k_{+}(s-t)-k(s)\right| d t
$$

converges for almost all $x$. We have

$$
\begin{aligned}
I(x)= & \int_{0}^{\varepsilon}|\varphi(x-s)| d s \int_{\varepsilon}^{\infty}\left|\ell^{\prime}(t)\right| \cdot|k(s)| d t \\
& +\int_{\varepsilon}^{\infty}|\varphi(x-s)| d s \int_{s}^{\infty}\left|\ell^{\prime}(t)\right| \cdot|k(s)| d t \\
& +\int_{\varepsilon}^{\infty}|\varphi(x-s)| d s \int_{\varepsilon}^{s}\left|\ell^{\prime}(t)\right| \cdot|k(s-t)-k(s)| d t \\
= & I_{1}+I_{2}+I_{3} .
\end{aligned}
$$

Obviously,

$$
I_{1}+I_{2} \leq c_{\varepsilon} \int_{0}^{\infty}|k(s) \varphi(x-s)| d s,
$$

where $c_{\varepsilon}=\int_{\varepsilon}^{\infty}\left|\ell^{\prime}(t)\right| d t$, which converges for almost all $x$ by Theorem 2.4. For the term $I_{3}$ it suffices to show the convergence of the integral

$$
\int_{\varepsilon}^{\infty}\left|\ell^{\prime}(t)\right| d t \int_{t}^{\infty}|\varphi(x-s)| \cdot(|k(s-t)|+|k(s)|) d s
$$

which has the form

$$
\int_{\varepsilon}^{\infty}\left|\ell^{\prime}(t)\right| g(x-t) d t+g(x) \int_{\varepsilon}^{\infty}\left|\ell^{\prime}(t)\right| d t
$$


with $g(x)=\int_{0}^{\infty}|k(s) \varphi(x-s)| d s \in L_{\Phi}\left(\mathbb{R}^{1}\right)$ by Theorem 2.4. It remains to note that the integrals in (5.17) converge.

We will show later in Lemma 5.4 that $N_{\varepsilon}(s)$ is the averaging kernel, so that the operator on the right-hand side of $(5.8)$ is the identity approximation operator.

\subsubsection{Properties of the averaging kernel $N_{\varepsilon}(x)$}

LEMmA 5.3. Let the Sonine kernel $k(x)$ satisfy assumptions (4.8) and (4.9). Beside formula (5.9), the kernel $N_{\varepsilon}(x)$ may also be represented in the following equivalent forms:

$$
\begin{aligned}
& N_{\varepsilon}(s)=\ell(\varepsilon)\left[k(s)-k_{+}(s-\varepsilon)\right]+\frac{d}{d s} \int_{\varepsilon}^{s} \ell(t) k_{+}(s-t) d t, \\
& N_{\varepsilon}(s)=\ell(\varepsilon)[k(s)-k(s-\varepsilon) v]-\int_{s-\varepsilon}^{s} \ell(s-t) k^{\prime}(t) d t, \quad s>\varepsilon, \\
& N_{\varepsilon}(s)=\ell(\varepsilon) k(s)+\int_{\varepsilon}^{s} \ell^{\prime}(t) k_{+}(s-t) d t, \\
& N_{\varepsilon}(s)= \begin{cases}\int_{0}^{\varepsilon} \ell^{\prime}(t)[k(s)-k(s-t)] d t, & s>\varepsilon, \\
k(s) \ell(\varepsilon), & 0<s<\varepsilon .\end{cases}
\end{aligned}
$$

Proof. From (5.9) we have (5.20). Relation (5.18) follows from (5.20) if we observe that

$$
\frac{d}{d s} \int_{0}^{s-\varepsilon} \ell(s-t) k(t) d t=\ell(\varepsilon) k(s-\varepsilon)+\int_{\varepsilon}^{s} \ell^{\prime}(t) k(s-t) d t .
$$

We prove (5.21). The second line in (5.21) is already contained in (5.18) and (5.20). Let $s>\varepsilon$. Then from (5.9) we have

$$
N_{\varepsilon}(s)=\int_{\varepsilon}^{s} \ell^{\prime}(t)[k(s-t)-k(s)] d t-\int_{s}^{\infty} \ell^{\prime}(t) k(s) d t+k(s) \ell(\infty)
$$

or

$$
\begin{aligned}
N_{\varepsilon}(s)= & \int_{0}^{s} \ell^{\prime}(t)[k(s-t)-k(s)] d t \\
& -\int_{0}^{\varepsilon} \ell^{\prime}(t)[k(s-t)-k(s)] d t+k(s) \ell(s)
\end{aligned}
$$

which turns into (5.21) by (4.2).

To prove (5.19), we use (5.21) and for $s>\varepsilon$ we have

$$
\begin{aligned}
N_{\varepsilon}(s) & =\lim _{\delta \rightarrow 0}\left[\int_{\delta}^{\varepsilon} \ell^{\prime}(t) k(s) d t-\int_{\delta}^{\varepsilon} \ell^{\prime}(t) k(s-t) d t\right] \\
& =\lim _{\delta \rightarrow 0}\left\{\ell(\delta)[k(s-\delta)-k(s)]+\ell(\varepsilon)[k(s)-k(s-\varepsilon)]-\int_{\delta}^{\varepsilon} \ell(t) k^{\prime}(s-t) d t\right\} .
\end{aligned}
$$


Since $k(s)$ is differentiable for $s>0$, the first term tends to zero as $\delta \rightarrow 0$, by (4.16), which yields (5.19).

LEMMA 5.4. If the Sonine kernel $k(x)$ satisfies assumptions (4.8) and (4.9), then $N_{\varepsilon}(x)$ has the properties of the identity approximation kernel

$$
\begin{gathered}
\sup _{0<\varepsilon<\varepsilon_{0}} \int_{0}^{\infty}\left|N_{\varepsilon}(x)\right| d x=M<\infty, \\
\lim _{\varepsilon \rightarrow 0} \int_{0}^{\infty} N_{\varepsilon}(t) d t=1,
\end{gathered}
$$

where $\int_{0}^{\infty} N_{\varepsilon}(t) d t \equiv 1$ in the case $k(\infty)=0$, and

$$
\lim _{\varepsilon \rightarrow 0} \int_{\delta}^{\infty}\left|N_{\varepsilon}(s)\right| d s=0, \quad \delta>0 .
$$

Proof. By (5.21), we have

$$
\int_{0}^{\infty}\left|N_{\varepsilon}(s)\right| d s=\ell(\varepsilon) \int_{0}^{\varepsilon}|k(s)| d s+\int_{\varepsilon}^{\infty}\left|N_{\varepsilon}(s)\right| d s
$$

for $0<\varepsilon<\varepsilon_{0}$. Here the first term is bounded for $0<\varepsilon \leq \varepsilon_{0}$ by (4.12). The second term is estimated by means of (5.19) as follows:

$$
\begin{aligned}
\int_{\varepsilon}^{\infty}\left|N_{\varepsilon}(s)\right| d s \leq & \ell(\varepsilon) \int_{\varepsilon}^{\infty}|k(s)-k(s-\varepsilon)| d s \\
& +\int_{\varepsilon}^{\infty} d s \int_{s-\varepsilon}^{s}\left|\ell(s-t) k^{\prime}(t)\right| d t .
\end{aligned}
$$

Here the first term is bounded by (4.24), while the second one is reduced after the change of order of integration to

$$
\int_{0}^{\varepsilon}\left|k^{\prime}(t)\right| d t \int_{\varepsilon-t}^{\varepsilon}|\ell(s)| d s+\int_{\varepsilon}^{\infty}\left|k^{\prime}(t)\right| d t \int_{0}^{\varepsilon} \ell(s) d s
$$

which is also uniformly bounded in view of (4.25) and (4.17).

To verify (5.27), we make use of representation (5.18). For the first term in (5.18) we have

$$
\begin{aligned}
\int_{0}^{\infty}\left[k(s)-k_{+}(s-\varepsilon)\right] d s & =\lim _{a \rightarrow \infty} \int_{0}^{a}\left[k(s)-k_{+}(s-\varepsilon)\right] d s \\
& =\lim _{a \rightarrow \infty} \int_{a-\varepsilon}^{a} k(s) d s=0
\end{aligned}
$$

by (4.21). To treat the second term in (5.18), we denote

$$
A_{\varepsilon}(s)=\int_{\varepsilon}^{s} \ell(t) k(s-t) d t, \quad s>\varepsilon,
$$

so that in view of (5.32),

$$
\int_{0}^{\infty} N_{\varepsilon}(s) d s=\int_{\varepsilon}^{\infty} \frac{d}{d s} A_{\varepsilon}(s) d s=A_{\varepsilon}(\infty) .
$$


It remains to show that $\lim _{\varepsilon \rightarrow 0} A_{\varepsilon}(\infty)=1$. We have

$$
\begin{aligned}
A_{\varepsilon}(\infty) & =\lim _{s \rightarrow \infty}\left[\int_{0}^{s} \ell(t) k(s-t) d t-\int_{0}^{\varepsilon} \ell(t) k(s-t) d t\right] \\
& =1-\lim _{s \rightarrow \infty} \int_{0}^{\varepsilon} \ell(t) k(s-t) d t .
\end{aligned}
$$

The remaining limit in (5.35) equals zero if $k(\infty)=0$, the limit existing by (4.10). Indeed, $\sup _{0<t<\varepsilon}|k(s-t)|=\left|k\left(s_{\varepsilon}\right)\right|$ with $s-\varepsilon<s_{\varepsilon}<s$, which tends to zero as $s \rightarrow \infty$. Hence $A_{\varepsilon}(\infty)=1$ and from (5.34) we obtain that $\int_{0}^{\infty} N_{\varepsilon}(t) d t=1$. In the case where $k(\infty) \neq 0$, we arrive at (5.27).

Finally, to prove (5.28), we make use of representation (5.19) taking into account that $\varepsilon<\delta<s$ and obtain

$$
\begin{aligned}
\lim _{\varepsilon \rightarrow 0} \int_{\delta}^{\infty}\left|N_{\varepsilon}(\xi)\right| d \xi \leq & \lim _{\varepsilon \rightarrow 0} \int_{\delta}^{\infty} \ell(\varepsilon)|k(s)-k(s-\varepsilon)| d s \\
& +\lim _{\varepsilon \rightarrow 0} \int_{\delta}^{\infty} d s \int_{0}^{\varepsilon} \ell(t)\left|k^{\prime}(s-t)\right| d t \\
= & : \lim _{\varepsilon \rightarrow 0} \ell(\varepsilon) I_{\varepsilon}^{1}+\lim _{\varepsilon \rightarrow 0} I_{\varepsilon}^{2} .
\end{aligned}
$$

The term $I_{\varepsilon}^{1}$ is estimated as in (4.28):

$$
\begin{aligned}
I_{\varepsilon}^{1} & \leq \int_{0}^{\infty}|k(x+\varepsilon)-k(x)| d x \leq \int_{0}^{\varepsilon} d t \int_{t+\delta}^{\infty}\left|k^{\prime}(x)\right| d x \\
& \leq \int_{0}^{\varepsilon} d t \int_{\delta}^{\infty}\left|k^{\prime}(x)\right| d x=c \varepsilon
\end{aligned}
$$

by (4.9). Then $\lim _{\varepsilon \rightarrow 0} \ell(\varepsilon) I_{\varepsilon}^{1}=0$ in view of (4.16).

For $I_{\varepsilon}^{2}$ we have

$$
I_{\varepsilon}^{2}=\int_{0}^{\varepsilon} \ell(t) d t \int_{\delta-t}^{\infty}\left|k^{\prime}(s)\right| d s \leq c(\delta) \int_{0}^{\varepsilon} \ell(t) d t \rightarrow 0,
$$

where $c(\delta)=\int_{\delta / 2}^{\infty}\left|k^{\prime}(s)\right| d s$ under the assumption that $\varepsilon \leq \delta / 2$.

6. Inversion theorem and characterization of the range $\mathbf{K}\left(L_{p}\right)$. Theorems 6.1 and 6.3 of this section are generalizations of [7, Theorems 6.1 and 6.2] which correspond to the special case $k(x)=x_{+}^{\alpha-1}$. The proof follows principally the same lines as in the proof in [7], but we emphasize that this generalization from the case of power kernel to the case of a general Sonine kernel required nontrivial efforts to show that in the general case the corresponding kernel in the integral representation (5.8) is an identity approximation kernel, see the proof of Lemmas 5.3 and 5.4 .

THEOREM 6.1. Let $k(x)$ be a Sonine kernel satisfying conditions (2.21), (4.8), and (4.9). Then

$$
\mathbf{K}^{-1} \mathbf{K} \varphi=\varphi, \quad \varphi \in L_{p}\left(\mathbb{R}^{1}\right), 1<p<\infty
$$


where

$$
\left(\mathbf{K}^{-1} f\right)(x)=\ell(\infty) f(x)+\lim _{\substack{\varepsilon \rightarrow 0 \\\left(L_{p}\right)}} \int_{\varepsilon}^{\infty}[f(x-t)-f(x)] \ell^{\prime}(t) d t .
$$

Proof. By Theorem 5.2, representation (5.8) is valid. Because of the properties of the kernel $N_{\varepsilon}(s)$ obtained in Lemma 5.4, Lemma 2.3 is applicable, with $X=L_{p}\left(\mathbb{R}^{n}\right)$, which completes the proof.

In Theorem 6.3 below we give a characterization of the range

$$
\mathbf{K}\left(L_{p}\right)=\left\{f: f(x)=(\mathbf{K} \varphi)(x), \varphi \in L_{p}(\mathbb{R})\right\}
$$

of the operator $\mathbf{K}$ under our general assumptions (2.21), (4.9), and (4.8) on $k(x)$. Naturally, this range is imbedded into a certain Orlicz space. In some cases it is possible to stay within the $L_{p}$-scale, namely under the assumption that there exists an $\alpha \in(0,1)$ such that the function $m(x)=x^{\alpha-1} k(x)$ satisfies the condition

$$
\sup _{\mathbb{R}_{+}^{1}}|m(x)|<\infty
$$

In the general case, dealing with the Orlicz space $L_{\Phi}$ with the Young function $\Phi$ defined by (2.22), we need to know that the function $\Phi$ has the $\Delta_{2}$-property because we will use Lemma 2.3 on identity approximation, see Remark 2.2. The following lemma is valid.

LEMMA 6.2. Let $k(x)$ satisfy assumptions (4.9) and (2.21). The Young function $\Phi(u)$ defined by (2.22) satisfies the $\Delta_{2}$-condition if and only if

$$
\varlimsup_{x \rightarrow 0} \frac{\int_{x}^{\infty} k^{*}(t)(x / t)^{1 / p} d t}{\int_{0}^{x} k(t) d t}<\infty .
$$

Proof. As is known, the $\Delta_{2}$-condition is equivalent to the condition

$$
\varlimsup_{u \rightarrow \infty}\left(\frac{u \Phi^{\prime}(u)}{\Phi(u)}\right)<\infty
$$

see [1, page 138], or equivalently,

$$
\varlimsup_{x \rightarrow \infty} \frac{\Phi^{-1}(x)}{x(d / d x) \Phi^{-1}(x)}<\infty .
$$

By (2.22) and (2.24) we have

$$
\begin{gathered}
\Phi^{-1}(x)=p\left[x^{1 / p-1} k^{* *}\left(\frac{1}{x}\right)+\int_{1 / x}^{\infty} \frac{k^{*}(s)}{s^{1 / p}} d s\right], \\
\frac{d}{d x} \Phi^{-1}(x)=x^{1 / p-2} k^{* *}\left(\frac{1}{x}\right),
\end{gathered}
$$

for $x \rightarrow \infty$. Then condition (6.7) reduces to (6.5). 
In Theorem 6.3 we denote for brevity

$$
Y\left(\mathbb{R}^{1}\right)= \begin{cases}L_{\Phi}\left(\mathbb{R}^{1}\right), & \text { if } k(x) \text { satisfies (2.21) and (6.5), } \\ L_{q}\left(\mathbb{R}^{1}\right), & \text { if } k(x) \text { satisfies (6.4), }\end{cases}
$$

where the Young function $\Phi(u)$ is defined by (2.22).

THEOREM 6.3. Let $k(x)$ satisfy conditions (2.21), (4.8), and (4.9). Then $f(x) \in$ $\mathbf{K}\left(L_{p}\right)$ if and only if $f(x) \in Y\left(\mathbb{R}^{1}\right)$ and one of the following conditions is satisfied:

$$
\lim _{\substack{\varepsilon \rightarrow 0 \\\left(L_{p}\right)}} \mathbf{K}_{\varepsilon}^{-1} f \in L_{p} \quad \text { or } \quad \sup _{\varepsilon>0}\left\|\mathbf{K}_{\varepsilon}^{-1} f\right\|_{L_{p}}<\infty .
$$

\section{PROOF}

NeCESSITY. Let $f=\mathbf{K} \varphi, \varphi \in L_{p}$. The statement $f(x) \in Y$ is immediate by the O'Neil and Hardy-Littlewood theorems. The existence of the limit $\lim _{\varepsilon \rightarrow 0} \mathbf{K}_{\varepsilon}^{-1} f$ is a consequence of Theorem 6.1. The uniform boundedness $\sup _{\varepsilon>0}\left\|\mathbf{K}_{\varepsilon}^{-1} f\right\|_{L_{p}}<\infty$ follows from representation (5.8) and property (5.26).

SuFFICIENCY. First we will show that, under the assumptions of the theorem, there exists a $\varphi \in L_{p}\left(\mathbb{R}^{1}\right)$ such that

$$
f(x)-f(x-h)=(\mathbf{K} \varphi)(x)-(\mathbf{K} \varphi)(x-h) \quad \forall h \in \mathbb{R}^{1} .
$$

We denote

$$
\left(A_{h} \varphi\right)(x)=\int_{-\infty}^{\infty} a_{h}(x-t) \varphi(t) d t=(\mathbf{K} \varphi)(x)-(\mathbf{K} \varphi)(x-h),
$$

where

$$
a_{h}(t)=k(t)-k_{+}(t-h) \in L_{1}\left(\mathbb{R}^{1}\right)
$$

by property (4.23).

We have

$$
A_{h} \mathbf{K}_{\varepsilon}^{-1} f=\mathbf{K}_{\varepsilon}^{-1} A_{h} f=\left(\mathbf{K}_{\varepsilon}^{-1} \mathbf{K} f\right)(x)-\left(\mathbf{K}_{\varepsilon}^{-1} \mathbf{K} f\right)(x-h)
$$

at least on "nice" functions; for example, on functions $f \in C_{0}^{\infty}$. Making use of representation (5.8), we arrive at the relation

$$
A_{h} \mathbf{K}_{\varepsilon}^{-1} f=\int_{0}^{\infty} N_{\varepsilon}(s)[f(x-t)-f(x-h-t)] d t
$$

at least for $f \in C_{0}^{\infty}$. The class $C_{0}^{\infty}$ is dense in the space $Y\left(\mathbb{R}^{1}\right)$; in the case $Y=L_{\Phi}$ it is valid under the $\Delta_{2}$-condition, see Lemma 2.3 and Remark 2.2, that is, under condition (6.5). Therefore, by boundedness of the operators in (6.15) we conclude that (6.15) is valid on the whole space $L_{p}\left(\mathbb{R}^{1}\right)$. 
Now we pass to the limit in (6.15) in $Y$-norm as $\varepsilon \rightarrow 0$, which is possible since the right-hand side of (6.15) converges to $f(x)-f(x-h)$ by Lemmas 5.4 and 2.3. We obtain

$$
f(x)-f(x-h)=\lim _{\varepsilon \rightarrow 0} A_{h} \mathbf{K}_{\varepsilon}^{-1} f
$$

with the limit treated in the sense of the space $Y\left(\mathbb{R}^{1}\right)$.

Let the first condition in (6.10) be satisfied. Then, by boundedness of the operator $A_{h}$ in $L_{p}$, there exists the limit

$$
\lim _{\substack{\varepsilon \rightarrow 0 \\(L p)}} A_{h} \mathbf{K}_{\varepsilon}^{-1} f=A_{h}\left(\lim _{\substack{\varepsilon \rightarrow 0 \\\left(L_{p}\right)}} \mathbf{K}_{\varepsilon}^{-1} f\right)=A_{h} \varphi \quad \text { with } \varphi=\lim _{\substack{\varepsilon \rightarrow 0 \\\left(L_{p}\right)}} \mathbf{K}_{\varepsilon}^{-1} f .
$$

Since $A_{h} \mathbf{K}_{\varepsilon}^{-1} f$ converges both in $Y$-norm and $L_{p}$-norm, the limit functions coincide almost everywhere and we arrive at relation (6.11). To arrive at (6.11) under the second condition in (6.10), we repeat the same arguments using a weak compactness of bounded sets in $L_{p}$, see details in [7, page 128] in the case $k(x)=x_{+}^{\alpha-1}$.

Relation (6.11) being obtained, it remains to observe that it immediately implies $f(x)=(\mathbf{K} \varphi)(x)$ since functions with equal differences may differ only by a constant which may be only zero in our case.

7. Sufficient conditions for a function to be a Sonine kernel and examples. Examples of kernels satisfying condition (1.2) given in [8] (see also [9]) are the following.

(1) Bessel-type functions:

$$
k(x)=(\sqrt{x})^{-v} J_{-v}(2 \sqrt{x}), \quad \ell(x)=(\sqrt{x})^{v-1} I_{v-1}(2 \sqrt{x})
$$

or symmetrically

$$
k(x)=(\sqrt{x})^{-v} I_{-v}(2 \sqrt{x}), \quad \ell(x)=(\sqrt{x})^{v-1} J_{v-1}(2 \sqrt{x}),
$$

where

$$
J_{v}(x)=\sum_{k=0}^{\infty} \frac{(-1)^{k}(x / 2)^{2 k+v}}{k ! \Gamma(k+v+1)}, \quad I_{v}(x)=\sum_{k=0}^{\infty} \frac{(x / 2)^{2 k+v}}{k ! \Gamma(k+v+1)}
$$

are the Bessel and modified Bessel functions, respectively, $0<v<1$. In particular, one may take

$$
k(x)=\frac{\cos (2 \sqrt{x})}{\sqrt{\pi x}}, \quad \ell(x)=\frac{\operatorname{ch}(2 \sqrt{x})}{\sqrt{\pi x}}
$$

or vice versa. 
(2) Power-exponential function and the incomplete gamma function:

$$
\begin{gathered}
k(x)=\frac{e^{-\lambda x}}{\Gamma(\alpha) x^{1-\alpha}} \\
\ell(x)=\lambda^{\alpha}\left[\frac{\alpha}{\Gamma(1-\alpha)} \Gamma(-\alpha, \lambda x)+1\right]=\lambda^{\alpha}+\frac{\alpha}{\Gamma(1-\alpha)} \int_{x}^{\infty} \frac{e^{-\lambda t}}{t^{1+\alpha}} d t
\end{gathered}
$$

where $0<\alpha<1, \lambda \geq 0$, and $\Gamma(-\alpha, x)$ is the incomplete gamma function (the associate kernel (7.6) was given in [8] in a different form).

Note that relation (1.2) in Laplace transforms $\mathscr{K}(s)=\int_{0}^{\infty} e^{-s t} k(t) d t$ has the form

$$
s \mathscr{K}(s) \mathscr{L}(s) \equiv 1
$$

Therefore, examples of Sonine kernels may be obtained via relation (7.7). For example, for (7.1) and (7.2) in view of (7.7), we may refer to the formula for the Laplace transform of the corresponding Bessel function:

$$
\int_{0}^{\infty} e^{-s x}(\sqrt{x})^{-v} J_{-v}(2 \sqrt{x}) d x=\frac{e^{-1 / s}}{s^{1-v}}
$$

see [3, formula 212.9.3]. For (7.5) and (7.6), by direct calculation of Laplace transforms we have

$$
\mathscr{K}(s)=\frac{1}{(\lambda+s)^{\alpha}}, \quad \mathscr{L}(s)=\frac{(\lambda+s)^{\alpha}}{s} .
$$

Some sufficient condition for a function $k(x)$ to be a Sonine kernel is given by Theorem 7.1 below. Let

$$
k(x)=\frac{a(x)}{x^{1-\alpha}}, \quad 0<\alpha<1, \quad a(x)=\sum_{k=0}^{\infty} a_{k} x^{k}, \quad a_{0} \neq 0 .
$$

THEOREM 7.1 (see [12]). There exists a unique analytic function $b(x)=$ $\sum_{k=0}^{\infty} b_{k} x^{k}$ such that the kernel $k(x)$ in (7.10) and the kernel

$$
\ell(x)=\frac{b(x)}{x^{\alpha}}
$$

are associate Sonine kernels; the series for $b(x)$ converges where the series for $a(x)$ converges. The coefficients $b_{k}$ may be uniquely determined from the following triangular algebraic system:

$$
\begin{gathered}
a_{0} b_{0}=\frac{\sin \alpha \pi}{\pi}, \\
\sum_{k=0}^{n} b_{k} a_{n-k} \Gamma(k+1-\alpha) \Gamma(\alpha+n-k)=0, \quad n \geq 1 .
\end{gathered}
$$


In [12], Theorem 7.1 was proved by means of contour integration in the complex plane. The reduction itself of Sonine condition (1.2) for functions (7.10) and (7.11) to the algebraic system (7.12) may be given in a direct form. Indeed, substituting (7.10) and (7.11) into (1.2), after the change of variables $t=x \xi$, we obtain

$$
\int_{0}^{1} \frac{1}{\xi^{\alpha}(1-\xi)^{1-\alpha}} \sum_{k=0}^{\infty} a_{k} x^{k}(1-\xi)^{k} \cdot \sum_{j=0}^{\infty} b_{j} x^{j} \xi^{j} d \xi \equiv 1 .
$$

Multiplying two power series and equalizing the coefficients, after easy calculations we arrive at (7.12).

Theorem 7.1 covers kernels close to power-type functions. Clearly, the class of Sonine kernels is wider and includes, for instance, functions with powerlogarithmic singularities at the origin. More precisely, functions of the form

$$
k(x)=h(x) x^{\alpha-1} \prod_{k=1}^{n}[\underbrace{\ln \cdots \ln }_{k} \frac{\gamma}{x}]^{v_{k}}
$$

are Sonine kernels, where $h(x)$ is any absolutely continuous function with $h(0) \neq 0,0<\alpha<1, v_{k} \in \mathbb{R}^{1}, k=2, \ldots, n, v_{1} \in \mathbb{Z}$ and $\gamma$ is a sufficiently large number, see [4] where a more general class of Sonine kernels was in fact considered, the reference to [6] is also relevant.

Examples given in (7.1), (7.2), (7.5), and (7.6) are of type of (7.10) and (7.11).

We mention also another example of the same nature:

$$
\begin{aligned}
& k(x)=x^{\alpha-1} \Phi(\beta, \alpha ;-\lambda x), \quad 0<\alpha<1, \\
& \ell(x)=\frac{\sin \alpha \pi}{\pi} x^{-\alpha} \Phi(-\beta, 1-\alpha ;-\lambda x),
\end{aligned}
$$

where $\Phi(\beta, \alpha ; z)=\sum_{k=0}^{\infty}\left((\beta)_{k} /(\alpha)_{k}\right)\left(z^{k} / k !\right)$ is the Kummer function (to see that these functions form associate Sonine kernels, we refer to [7, formulas (37.1) and (37.31)])

We also dwell on examples not covered by Theorem 7.1, namely

$$
\begin{aligned}
& k(x)=1+\frac{a}{\sqrt{x}}, \quad a>0, \\
& k(x)=1-\frac{a}{x^{1-\alpha}}, \quad a>0 .
\end{aligned}
$$

In the case of (7.16) we have

$$
\ell(x)=\frac{1}{\sqrt{\pi x}}-b e^{b^{2} x} \operatorname{erfc}(b \sqrt{x}), \quad b=a \Gamma(\alpha),
$$

which may be verified via Laplace transforms:

$$
\mathscr{K}(s)=\frac{\sqrt{s}+b}{s}, \quad \mathscr{L}(s)=\frac{1}{\sqrt{s}+b} .
$$


We observe that the function (7.18) satisfies the property $\ell^{\prime}(x) \in L_{1}(\varepsilon, \infty)$, $\varepsilon>0$, which may be obtained by means of the known asymptotics for the error function:

$$
\operatorname{erfc} x=\frac{e^{-x^{2}}}{\sqrt{\pi}}\left(\frac{1}{2}-\frac{1}{2 x^{3}}+\frac{3}{4 x^{5}}-\frac{15}{8 x^{7}}+\cdots\right) \text { as } x \rightarrow \infty \text {. }
$$

For the function $k(x)$ in (7.17) the associate Sonine kernel is the generalized Mittag-Leffler function:

$$
\ell(x)=b x^{-\alpha} E_{1-\alpha, 1-\alpha}\left(b x^{1-\alpha}\right), \quad b=a \Gamma(\alpha),
$$

where $E_{\alpha, \beta}(x)=\sum_{k=0}^{\infty}\left(x^{k} / \gamma(\alpha k+\beta)\right)$, which may be also verified by means of Laplace transforms, see [7, formula (1.93)]. However, this kernel and its derivatives grow exponentially as $x \rightarrow \infty$.

Finally, we mention a kernel of power-logarithmic type

$$
k(x)=\frac{\ln (1 / x)+A}{\Gamma(\alpha) x^{1-\alpha}},
$$

where $A \in \mathbb{R}^{1}$, the associate Sonine kernel is expressed in terms of the special function known as Volterra function:

$$
\ell(x)=\mu_{\alpha, h}(x), \quad h=\frac{\Gamma^{\prime}(\alpha)}{\Gamma(\alpha)}-A,
$$

where

$$
\mu_{\alpha, h}(x)=\int_{0}^{\infty} \frac{x^{t-\alpha} e^{h t}}{\Gamma(t+1-\alpha)} d t
$$

Relation (1.2), in the case of (7.22) and (7.23), is a consequence of the formula

$$
\int_{0}^{\infty} e^{-s x} \mu_{\alpha, h}(x) d x=\frac{s^{\alpha-1}}{\ln s-h}, \quad s>e^{h},
$$

which can be checked directly. The function $\mu_{\alpha, h}$ and the corresponding integral equation of the first kind with power-logarithmic kernel were first studied in [10], see also [11]; closed form solvability of power-logarithmic integral equations of the first kind of more general form can be found in [7, Section 32].

From the asymptotics of the Volterra function at the origin, see [10], or [7, formula (32.8)], it follows that $\ell^{\prime}(x)=\mu_{\alpha+1, h}(x)$ is negative near the origin and then $\ell(x)=\mu_{\alpha, h}(x)$ satisfies the monotonicity condition (4.8). However, the function $\mu_{\alpha, h}(x)$ is exponentially growing at infinity (like $e^{x e^{h}}$ ) and the same is for its derivative.

As some of the above examples show, the associate Sonine kernel $\ell(x)$ may grow at infinity and even exponentially, although the kernel $k(x)$ was decreasing, and the condition $\ell^{\prime}(x) \in L_{1}(\varepsilon, \infty)$ is not satisfied. Therefore, the general inversion formula (6.2) is not applicable in this case. However, it may be 
modified in such a way that it is applicable to functions $f(x)$ with support in $(-N, \infty), N<\infty$. This modification for a general Sonine-type kernel will be realized in another paper. In this relation, see [5] where a finite interval analogue of the Marchaud formula, that is, formula (5.4) for the kernel (7.22) was obtained.

Formula (6.2) in its direct form is applicable, for example, to kernels (7.5), (7.6), and (7.16).

In the case of (7.5) and (7.6) we note that the inversion formula obtained by Theorem 6.1 for the equation

$$
\frac{1}{\Gamma(\alpha)} \int_{-\infty}^{x} e^{-\lambda(x-t)}(x-t)^{\alpha-1} \varphi(t) d t=f(x) \text { for } \varphi(x) \in L_{p}\left(\mathbb{R}^{1}\right), 1 \leq p<\infty,
$$

has the form

$$
\varphi(x)=\lambda^{\alpha} f(x)+\frac{\alpha}{\Gamma(1-\alpha)} \lim _{\substack{\varepsilon \rightarrow 0 \\\left(L_{p}\right)}} \int_{\varepsilon}^{\infty} \frac{f(x)-f(x-t)}{t^{1+\alpha}} e^{-\lambda t} d t
$$

which formally coincides with

$$
\varphi(x)=\frac{\alpha}{\Gamma(1-\alpha)} \lim _{\substack{\varepsilon \rightarrow 0 \\\left(L_{p}\right)}} \int_{\varepsilon}^{\infty} \frac{f(x)-f(x-t) e^{-\lambda t}}{t^{1+\alpha}} d t
$$

since $\int_{0}^{\infty}\left(1-e^{-t} / t^{1+\alpha}\right) d t=\Gamma(1-\alpha) / \alpha$ (see [7, formula (5.81)]); see [7, page 335 ] about construction (7.28). However, the form of inversion (7.28) assumes that we have to look for solutions such that $e^{\lambda t} \varphi(t) \in L_{p}\left(\mathbb{R}^{1}\right)$.

Appendix. The Chebyshev inequality (2.1) without the assumption of the integrability of the product $k(x) \ell(x)$ on $[0, \varepsilon]$ is proved via the same ideas as in the case of integrability if we first cut off the origin. Namely, following the standard proof, see, for example, [13], we observe that

$$
\int_{\delta}^{x-\delta} \int_{\delta}^{x-\delta}[k(x-t)-k(y)][\ell(x-t)-\ell(y)] d t d y
$$

is positive if $k(x)$ and $\ell(x)$ have monotonicity of the same sign, and is negative if they gave monotonicity of an opposite sign. Hence we obtain

$$
(x-2 \delta) \int_{\delta}^{x-\delta} k(t) \ell(t) d t \geq \int_{\delta}^{x-\delta} k(t) d t \int_{\delta}^{x-\delta} \ell(t) d t
$$


in the former case, the sign of the inequality being opposite in the latter case. When $k(x)$ and $\ell(x)$ have the same monotonicity, the functions $k(x-t)$ and $\ell(t)$ have the opposite monotonicity; therefore,

$$
(x-2 \delta) \int_{\delta}^{x-\delta} k(x-t) \ell(t) d t \leq \int_{\delta}^{x-\delta} k(x-t) d t \int_{\delta}^{x-\delta} \ell(t) d t
$$

and it remains to pass to the limit as $\delta \rightarrow 0$ to obtain (2.1).

\section{REFERENCES}

[1] A. Kufner, O. John, and S. Fučík, Function Spaces, Noordhoff International Publishing, Leyden, 1977.

[2] R. O. O'Neil, Fractional integration in Orlicz spaces. I, Trans. Amer. Math. Soc. 115 (1965), no. 3, 300-328.

[3] A. P. Prudnikov, Yu. A. Brychkov, and O. I. Marichev, Integrals and Series. Vol. 2. Special Functions, 2nd ed., Gordon \& Breach Science Publishers, New York, 1988, translated from the Russian by N. M. Queen.

[4] B. S. Rubin, An imbedding theorem for images of convolution operators on a finite segment, and operators of potential type. I, Izv. Vyssh. Uchebn. Zaved. Mat. (1982), no. 1, 53-63 (Russian).

[5] _ An analogue of the Marchaud derivative for convolutions with powerlogarithm kernels on a finite interval, Mathematical Analysis and Its Applications, Rostov. Gos. Univ., Rostov, 1992, pp. 133-139 (Russian).

[6] B. S. Rubin and G. F. Volodarskaja, An imbedding theorem for convolutions on a finite interval and its application to integral equations of the first kind, Dokl. Akad. Nauk SSSR 244 (1979), no. 6, 1322-1326 (Russian), translation in Soviet Math. Dokl., 20 (1979), no. 1, 234-239.

[7] S. G. Samko, A. A. Kilbas, and O. I. Marichev, Fractional Integrals and Derivatives. Theory and Applications, Gordon and Breach Science Publishers, Yverdon, 1993, translated from Russian edition, Fractional Integrals and Derivatives and Some of Their Applications, Nauka i Tekhnika, Minsk, 1987.

[8] N. Sonine, Sur la généralisation d'une formule d'Abel, Acta Math. 4 (1884), 171176 (French).

[9] _ Investigations of Cylinder Functions and Special Polynomials, Gos. Izdat. Tekhn. Liter., Moscow, 1954 (Russian), pp. 244.

[10] V. Volterra, Teoria delle potenze dei logaritmi e delle funzioni di composizione, Atti Accad. dei Lincei, Ser. 511 (1916), 167-249 (Italian).

[11] V. Volterra and J. Pérés, Leçons sur la Composition et les Fonctions Permutables, Gauthier-Villars, Paris, 1924 (French).

[12] J. Wick, Über eine Integralgleichung vom Abelschen Typ, Z. Angew. Math. Mech. 48 (1968), no. 8, T39-T41 (German).

[13] A. Ya. Yakubov and L. D. Shankishvili, Some inequalities for convolution integral transforms, Integral Transform. Spec. Funct. 2 (1994), no. 1, 65-76.

Stefan G. Samko: Faculdade de Ciencias e Tecnologia, Universidade do Algarve, Campus de Gambelas, Faro 8000, Portugal

E-mail address: ssamko@ua7g.pt

URL: http://w3.ualg.pt/ ssamko/

Rogério P. Cardoso: Avenida 25 de Abril, Lote 16, 5 Esq., 8500-610 Portimo, Portugal

E-mail address: rpcardoso@netcabo.pt 


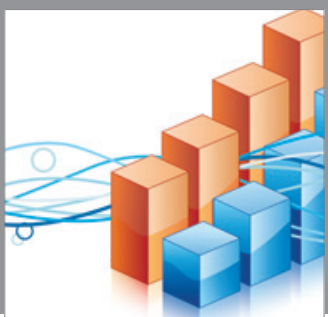

Advances in

Operations Research

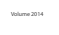

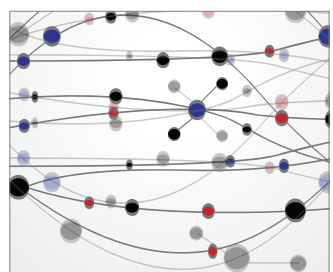

\section{The Scientific} World Journal
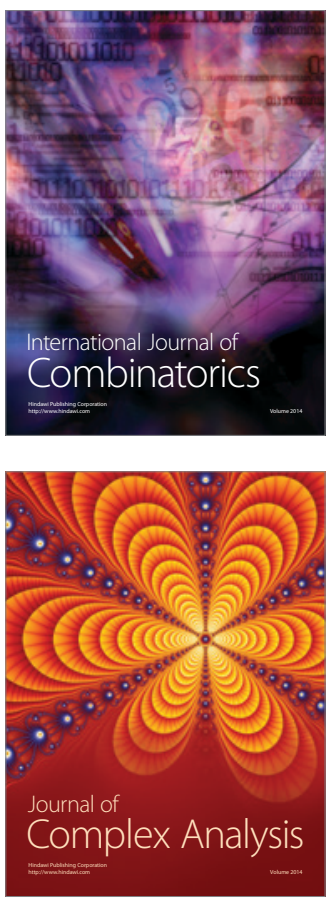

International Journal of

Mathematics and

Mathematical

Sciences
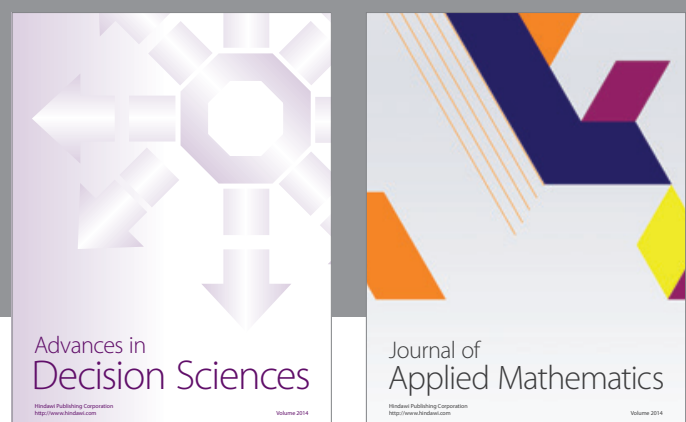

Journal of

Applied Mathematics
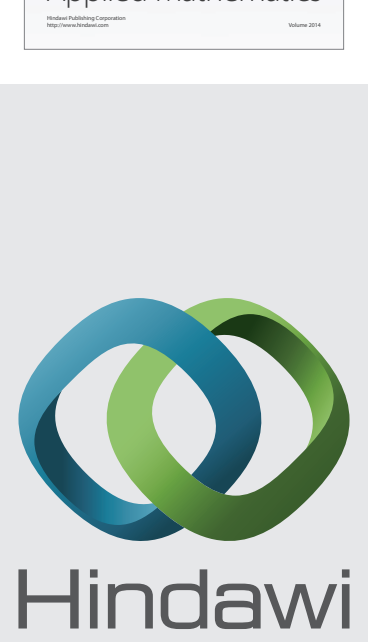

Submit your manuscripts at http://www.hindawi.com
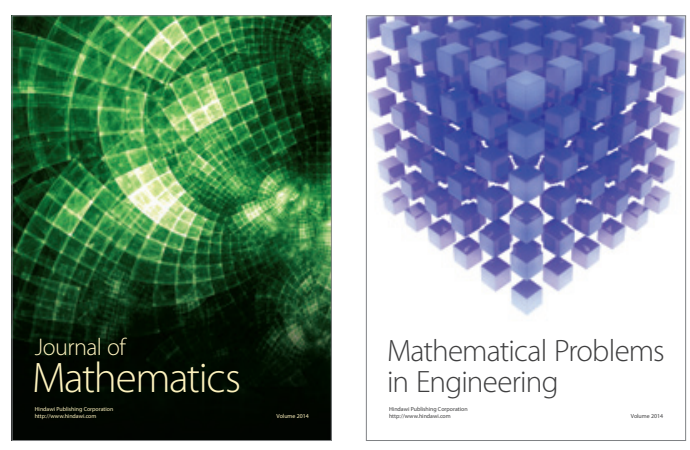

Mathematical Problems in Engineering
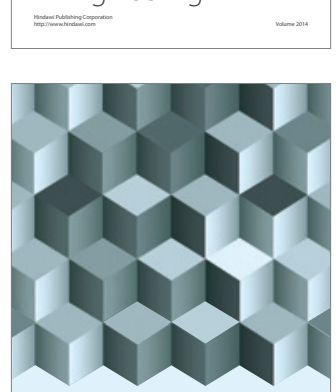

Journal of

Function Spaces
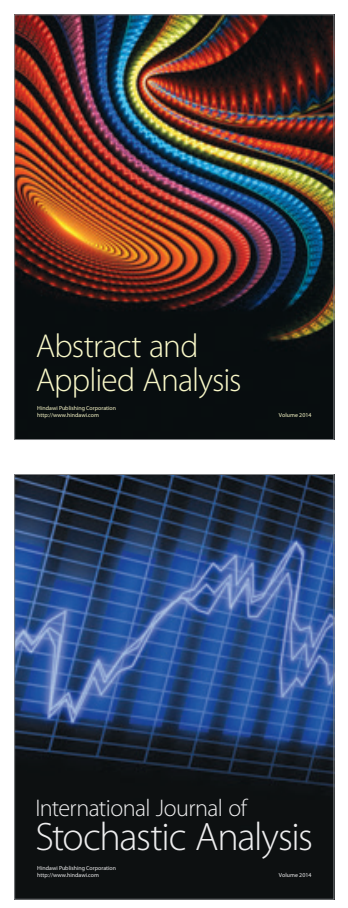

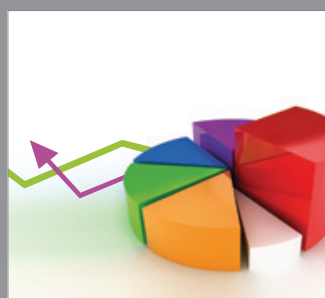

ournal of

Probability and Statistics

Promensencen
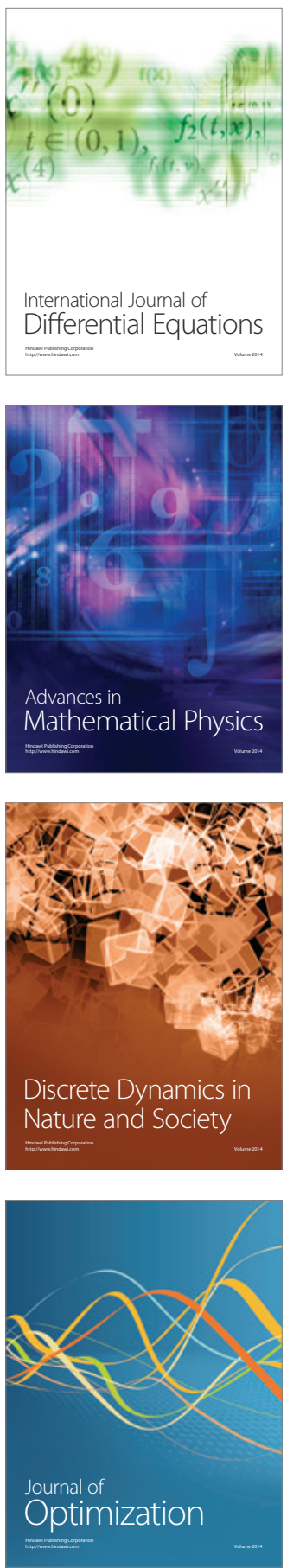